\title{
Clinical Study \\ Effects of Ropivacaine in Patient-Controlled Epidural Analgesia on Uterine Electromyographic Activities during Labor
}

\author{
Xueya Qian, ${ }^{1}$ Qingning Wang, ${ }^{2}$ Xinxu Ou, ${ }^{1}$ Pin Li, ${ }^{1}$ Baisong Zhao, ${ }^{2}$ and Huishu Liu $\mathbb{D}^{1}$ \\ ${ }^{1}$ Department of Obstetrics, Guangzhou Women and Children's Medical Center, Guangzhou Medical University, Guangzhou, China \\ ${ }^{2}$ Department of Anesthesia, Guangzhou Women and Children's Medical Center, Guangzhou Medical University, Guangzhou, China \\ Correspondence should be addressed to Huishu Liu; huishuliu@hotmail.com
}

Received 25 July 2018; Accepted 25 October 2018; Published 7 November 2018

Academic Editor: Mittal Suneeta

Copyright (c) 2018 Xueya Qian et al. This is an open access article distributed under the Creative Commons Attribution License, which permits unrestricted use, distribution, and reproduction in any medium, provided the original work is properly cited.

\begin{abstract}
Epidural analgesia is effective in relieving pain during labor. However, concerns as to compromised labor progress and outcomes arise. This study aimed to assess the effect of patient-controlled epidural analgesia (PCEA) with ropivacaine on uterine electromyography (EMG) activities and outcomes in labor. A total of 213 pregnant women were divided into three groups: the PCEA with ropivacaine group $(n=78)$, the PCEA with levobupivacaine group $(n=66)$, and a control group that did not receive PCEA $(n=69)$. Uterine EMG activities were recorded during the first stage of labor. Maternal and fetal outcomes also were assessed. The primary outcomes of this study were EMG activities. No significant differences were observed in patient demographics or neonatal weight among the three groups. Compared to the PCEA with levobupivacaine group, the control and PCEA with ropivacaine groups had lower rates of oxytocin administration $(\mathrm{P}<0.05)$ and shorter durations of the first stage of labor $(\mathrm{P}<0.05)$. For the EMG activities, the PCEA with ropivacaine group showed a higher power $(\mathrm{P}<0.01)$ and higher peak frequency $(\mathrm{P}<0.05)$ than the $\mathrm{PCEA}$ with levobupivacaine group. With ropivacaine, the EMG activities remained stable 30-120 min. Compared with levobupivacaine, the use of ropivacaine in PCEA has no suppressive effect on uterine EMG activities during the first stage of labor. In addition, ropivacaine leads to labor progress and delivery outcomes similar to those in the control group, as well as similar and favorable analgesic satisfaction with the use of levobupivacaine.
\end{abstract}

\section{Introduction}

Patient-controlled epidural analgesia (PCEA) is a well-accepted technique for pain relief during labor. However, there are still concerns that epidural labor analgesia may lead to prolongation of labor [1,2], malposition of the fetal head [3], increased use of oxytocin $[4,5]$, and even increased instrumental deliveries $[5,6]$. These effects may be due to the direct inhibition of the myometrial contractions by local anesthetics during labor [7-9].

Ropivacaine has been used as obstetric anesthesia because it offers good analgesic properties without causing significant motor blockade or systemic toxicity [10-12]. However, whether the use of ropivacaine affects uterine contractions is largely unknown. An important reason may be the lack of an objective and precise method for evaluating the effect of PCEA on myometrial contraction during labor. Recently, uterine electromyography (EMG) has emerged as a useful method for monitoring the excitability and contractility of the myometrium [13-15]. Our previous study indicated that uterine EMG was valuable for assessing uterine muscle activities during labor [16]. We also found that PCEA with levobupivacaine suppressed uterine EMG activities and prolonged the first stage of labor [17]. Considering the advantage of ropivacaine without significant motor blockade during analgesia, we hypothesized that PCEA with ropivacaine could have less inhibitory effects on uterine EMG activities compared to levobupivacaine during labor. The objective of this study was to investigate uterine EMG activities and labor outcomes in patients receiving PCEA with ropivacaine.

\section{Material and Methods}

2.1. Patients. This prospective cohort study included 213 patients at the Guangzhou Women and Children's Medical Center treated between 2015 and 2018. The study protocol 
was approved by the institutional review board (protocol No. 2014110533) and registered at ClinicalTrials.gov (registration No. NCT02036242). Patients were included in this study if they met the following inclusion criteria: (1) singleton pregnancy, age $\leq 35$ years, and a gestational age of 37-42 weeks; and (2) fetus with cephalic presentation. According to the standard obstetric indications, oxytocin was titrated by the obstetricians. The completed gestational weeks were determined by the date of the first day of the last menstrual period and confirmed by an ultrasound scan. Women with medical complications of pregnancy that required delivery via cesarean section or interventions that could influence the labor duration were excluded.

Patients were divided into three groups: those who received PCEA with levobupivacaine (levobupivacaine group, $\mathrm{n}=66$ ), those who received PCEA with ropivacaine (ropivacaine group, $n=78$ ), and those who did not receive PCEA (control group, $n=69$ ) nor other types of analgesia. Written informed consent was obtained from all patients. In our practice, whether or not PCEA treatment was administered was based on the patient's choice, and the use of levobupivacaine or ropivacaine was decided by the anesthesiologists.

According to the different time points of EMG recording, patients receiving PCEA with ropivacaine were subdivided into three groups: $30 \mathrm{~min}(\mathrm{n}=26), 60 \mathrm{~min}(\mathrm{n}=27)$, and 120 $\min (\mathrm{n}=25)$.

2.2. PCEA Protocol. After epidural catheter placement into the L2-L3 interspace, patients in the two PCEA groups received a first analgesic drug of $0.0625 \%$ ropivacaine and $0.0625 \%$ levobupivacaine in $10 \mathrm{ml}$ saline, as well as $5 \mu \mathrm{g}$ sufentanil. Patients were then placed in the supine position with left uterine displacement. A PCEA device containing $0.0625 \%$ ropivacaine or levobupivacaine and $0.4 \mu \mathrm{g} / \mathrm{ml}$ sufentanil in $240 \mathrm{ml}$ saline was started, with a background infusion rate of $6 \mathrm{ml} / \mathrm{h}$ and a bolus dose rate of $8 \mathrm{ml}$ for a lockout time of $15 \mathrm{~min}$. An anesthesiologist who was unaware of the grouping condition scored and recorded the visual analog scale (VAS) score (0-10) for pain reported by the patients at 0 , 15 , and 30 min after PCEA catheterization. Oxytocin was only administered after labor if needed. Patient characteristics including age, body mass index (BMI), gestational age, and oxytocin administration were recorded. Maternal and fetal outcomes including neonatal weight, duration of the first stage of labor, blood loss within $2 \mathrm{~h}$ after placenta delivery, Apgar scores at 1, 5, and $10 \mathrm{~min}$, and incidence of meconiumstained amniotic fluid were also recorded.

2.3. EMG Recording. Uterine EMG data were collected noninvasively from the abdominal surface using the PowerLab electromyographic instrumentation (AD Instruments, Castle Hill, Australia). In detail, four Ag-AgCl Beckman differential bipolar electrodes (Jun Kang Medical Supplies, Shanghai, China) were placed around the navel, with each electrode separated from the other by a distance of $3 \mathrm{~cm}$. A reference electrode was attached on the hip of the patient.

A specific filter with a band-pass of $0.34-1.00 \mathrm{~Hz}$ was used to exclude interference signal components during EMG recording [18-20]. Using an external tocodynamometry (TOCO, Sunray, Guangzhou, China) connected with the PowerLab and a standard maternal-fetal monitor (Philips, Avalon FM20, Best, The Netherlands), patients were continuously monitored for $30 \mathrm{~min}$. EMG activity recordings included the duration of burst, number of burst, root mean square (RMS), power, and peak frequency.

2.4. EMG Analysis. As described in our previous studies, standardized criteria were used to identify uterine EMG bursts: (1) a set of positive signals with an amplitude twice the baseline values; (2) a set of signals not returning to the baseline within 15 seconds; and (3) a burst often accompanied by contractions displayed on the TOCO $[16,21]$. To assess the signal stability, uterine EMG bursts were analyzed $10 \mathrm{~min}$ after the beginning of the recording as a standard procedure. One trained investigator who was experienced in EMG procedures performed the EMG and analyzed the data using Chart 8.0 software (ADInstruments, Castle Hill, Australia).

2.5. Statistical Analysis. Statistical analyses were performed using SPSS software (version 22.0; IBM SPSS Inc., Chicago, IL). On sample size calculations, 47 subjects per group were required with a power of 0.90 and an alpha of 0.05 based on our previous studies $[16,21]$. Continuous variables were compared using one-way analysis of variance or KruskalWallis test when appropriate for pairwise comparisons and because of non-normal distribution of variables. Categorical variables were analyzed using the Chi-squared test. Twosided P-values $<0.05$ were considered statistically significant.

\section{Results}

3.1. Patient Characteristics and Labor Outcomes. As shown in Table 1, patients in the three groups had similar demographic characteristics. The percentages of patients who needed oxytocin administration after labor were significantly lower in the control and ropivacaine groups compared to the levobupivacaine group $(\mathrm{P}=0.02)$.

Table 2 describes the maternal and fetal outcomes. The duration of the first stage of labor was significantly shorter in the control and ropivacaine groups than in the levobupivacaine group. Patients in the ropivacaine group had higher Apgar scores at 1 and $5 \mathrm{~min}(\mathrm{P}<0.001)$ compared to those in the other two groups. All patients in the three groups had similar labor outcomes including cesarean delivery rate, instrumental delivery rate, and postpartum hemorrhage.

3.2. Comparison of Analgesic Effects. As shown in Figure 1, patients in both groups were in severe pain at the time when labor analgesia was administered. The VAS scores of the two groups decreased significantly over time $(\mathrm{P}<0.05)$ but did not differ significantly between the two groups at the same time point $(\mathrm{P}>0.05)$. It was suggested that when levobupivacaine and ropivacaine were used in PCEA, they both took effect within 15 minutes and achieved a good analgesic effect within 30 minutes. Consistently, no difference was observed in the analgesic effect of the two drugs. 
TABLE 1: Patient characteristics.

\begin{tabular}{|c|c|c|c|c|}
\hline & $\begin{array}{c}\text { Control } \\
n=69\end{array}$ & $\begin{array}{c}\text { Levobupivacaine } \\
n=66\end{array}$ & $\begin{array}{c}\text { Ropivacaine } \\
\mathrm{n}=78\end{array}$ & $P$ value \\
\hline Age (years) & $27.99 \pm 0.38$ & $28.74 \pm 0.36$ & $28.94 \pm 0.36$ & 0.16 \\
\hline BMI $\left(\mathrm{kg} / \mathrm{m}^{2}\right)$ & $25.23 \pm 0.31$ & $25.27 \pm 0.28$ & $26.17 \pm 0.40$ & 0.09 \\
\hline Gestational age (weeks) & $39.40 \pm 0.13$ & $39.69 \pm 0.12$ & $39.58 \pm 0.11$ & 0.23 \\
\hline Multipara, n (\%) & $7(10.15 \%)$ & $9(13.64 \%)$ & $8(10.26 \%)$ & 0.77 \\
\hline Oxytocin administration, $\mathbf{n}(\%)$ & $18(26.09 \%)^{\mathrm{a}}$ & $31(46.97 \%)$ & $22(28.21 \%)^{\mathrm{a}}$ & 0.02 \\
\hline
\end{tabular}

BMI: body mass index. Data are presented as mean \pm standard error of the mean, $\mathrm{n}(\%)$, or median (range).

Table 1 shows the patient characteristics of the three groups. The oxytocin administration rates in the control and ropivacaine groups were significantly lower than that in the levobupivacaine group $(\mathrm{P}=0.02)$.

${ }^{\mathrm{a}} \mathrm{P}<0.05$ vs levobupivacaine group.

TABLE 2: Maternal and fetal outcomes.

\begin{tabular}{|c|c|c|c|c|}
\hline & $\begin{array}{c}\text { Control } \\
\mathrm{n}=69\end{array}$ & $\begin{array}{l}\text { Levobupivacaine } \\
\qquad \mathrm{n}=66\end{array}$ & $\begin{array}{c}\text { Ropivacaine } \\
\mathrm{n}=78\end{array}$ & $P$ value \\
\hline Cesarean, n (\%) & $4(5.80 \%)$ & $10(15.15 \%)$ & $7(8.97 \%)$ & 0.18 \\
\hline Instrumental, $\mathbf{n}(\%)$ & $7(10.15 \%)$ & $5(7.58 \%)$ & $5(6.41 \%)$ & 0.70 \\
\hline Neonatal birth weight (g) & $3148.33 \pm 33.81$ & $3215.53 \pm 39.58$ & $3272.31 \pm 44.90$ & 0.08 \\
\hline Duration of 1st stage of labor (min) & $562.00 \pm 25.06^{\mathrm{b}}$ & $677.14 \pm 32.36$ & $590.63 \pm 25.41^{\mathrm{b}}$ & 0.01 \\
\hline Postpartum hemorrhage (ml) & $252.46 \pm 10.09$ & $247.73 \pm 8.93$ & $245.38 \pm 10.99$ & 0.88 \\
\hline 1 minute Apgar & $9(8-10)$ & $9(7-10)$ & $9(6-10)^{\mathrm{a}}$ & $<0.001$ \\
\hline 5 minute Apgar & $9(8-10)$ & $9(8-10)$ & $9(9-10)^{\mathrm{a}}$ & $<0.001$ \\
\hline 10 minute Apgar & $9(9-10)$ & $9(8-10)$ & $9(8-10)$ & 0.98 \\
\hline Meconium stained amniotic fluid, n (\%) & $9(13.04 \%)$ & $12(18.18 \%)$ & $18(23.38 \%)$ & 0.29 \\
\hline
\end{tabular}

Data are presented as mean \pm standard error of the mean, $\mathrm{n}(\%)$, or median (range).

Table 2 shows maternal and fetal outcomes in the three groups. The episiotomy rate $(\mathrm{P}=0.001)$ and Apgar scores at 1 and 5 min $(\mathrm{P}<0.001)$ in the ropivacaine group were significantly higher than those in the levobupivacaine and control groups. Both the control and ropivacaine groups had a shorter duration of first stage of labor than that described in the levobupivacaine group $(\mathrm{P}=0.01)$.

${ }^{\mathrm{a}} \mathrm{P}<0.001$ vs control or levobupivacaine group.

${ }^{\mathrm{b}} \mathrm{P}<0.05$ vs levobupivacaine group.

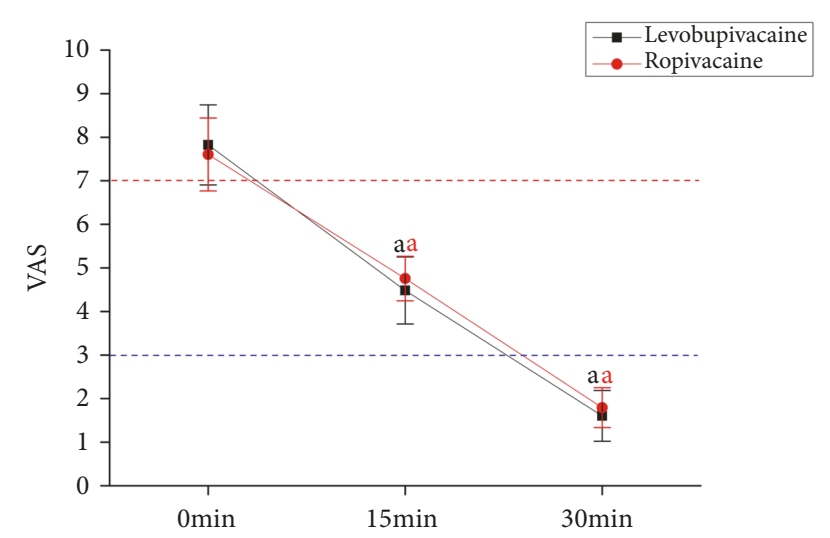

FIGURE 1: Analgesic effects of levobupivacaine and ropivacaine. VAS: visual analog scale for pain. The VAS score did not differ statistically between the two groups at the same time points. The horizontal red and blue lines indicate 7 and 3 on the VAS scale, respectively (7-10 for severe pain, 4-6 for moderate pain, and 0-3 for mild pain).

3.3. EMG Activities. Representative EMG image of the patient in ropivacaine group is shown in Figure 2. The results for uterine EMG activities are shown in Table 3. The two PECA groups had a significantly lower RMS compared to the control group $(\mathrm{P}<0.001$; Figure $3(\mathrm{a}))$. Both the control and ropivacaine groups had a higher power than the levobupivacaine group $(P=0.005$; Figure $3(b))$. The peak frequency was significantly higher in the ropivacaine group than in either of the other two groups (Figure 3(c)). There were no significant differences in the duration or the number of bursts among the three groups $(\mathrm{P}>0.05)$.

Regarding the EMG results at 30,60, and $90 \mathrm{~min}$ in the ropivacaine group, there was no significant difference in uterine EMG activity among the three different time points (Table 4).

\section{Discussion}

In this study, we assessed uterine EMG activities and obstetric outcomes during the first stage of labor with different labor analgesia regimens. The results suggest that (1) ropivacaine use had no inhibitory effect on uterine contractions compared with levobupivacaine; (2) ropivacaine use did not prolong the labor progress or impact delivery outcomes; and (3) ropivacaine use produced a comparable analgesic effect to that of levobupivacaine for epidural labor analgesia. 
TABLE 3: Uterine EMG activities in three groups.

\begin{tabular}{|c|c|c|c|c|}
\hline & $\begin{array}{c}\text { Control } \\
n=69\end{array}$ & $\begin{array}{c}\text { Levobupivacaine } \\
n=66\end{array}$ & $\begin{array}{c}\text { Ropivacaine } \\
n=78\end{array}$ & $P$ value \\
\hline Duration (s) & $44.92 \pm 2.15$ & $52.04 \pm 3.30$ & $49.35 \pm 1.46$ & 0.66 \\
\hline Number of bursts (n) & $3.65 \pm 0.13$ & $3.64 \pm 0.14$ & $3.49 \pm 0.07$ & 0.05 \\
\hline RMS (mV) & $0.09 \pm 0.01^{\mathrm{a}}$ & $0.05 \pm 0.004$ & $0.06 \pm 0.01$ & $<0.001$ \\
\hline Power $\left(\mathrm{nV}^{2}\right)$ & $7.69 \pm 1.37^{\mathrm{b}}$ & $3.42 \pm 0.59$ & $6.19 \pm 1.31^{\mathrm{b}}$ & 0.005 \\
\hline Peak frequency $(\mathrm{Hz})$ & $0.43 \pm 0.01$ & $0.42 \pm 0.01$ & $0.45 \pm 0.01^{\mathrm{c}}$ & 0.03 \\
\hline
\end{tabular}

RMS: root mean square. Data are presented as mean \pm standard error of the mean.

Table 3 shows uterine EMG activities of the three groups. The RMS $(\mathrm{P}<0.001)$ in the control group was significantly higher than those in the levobupivacaine and ropivacaine groups. Both the control and ropivacaine groups had a higher power than the levobupivacaine group $(\mathrm{P}=0.005)$. There were no significant differences in the duration or number of bursts among the groups $(\mathrm{P}>0.05)$.

${ }^{\mathrm{a}} \mathrm{P}<0.001$ vs levobupivacaine or ropivacaine group.

${ }^{\mathrm{b}} \mathrm{P}<0.05$ vs levobupivacaine group.

${ }^{\mathrm{c}} \mathrm{P}<0.05$ vs control or levobupivacaine group.

TABLE 4: Uterine EMG activities in the ropivacaine group.

\begin{tabular}{|c|c|c|c|c|}
\hline & $\begin{array}{c}30 \mathrm{~min} \\
\mathrm{n}=26\end{array}$ & $\begin{array}{c}60 \mathrm{~min} \\
\mathrm{n}=27\end{array}$ & $\begin{array}{c}120 \mathrm{~min} \\
\mathrm{n}=25\end{array}$ & $P$ value \\
\hline Duration (s) & $46.29 \pm 2.37$ & $49.65 \pm 2.58$ & $52.22 \pm 2.56$ & 0.26 \\
\hline Number of bursts (n) & $3.31 \pm 0.13$ & $3.48 \pm 0.11$ & $3.68 \pm 0.11$ & 0.09 \\
\hline RMS (mV) & $0.06 \pm 0.01$ & $0.06 \pm 0.01$ & $0.06 \pm 0.01$ & 0.97 \\
\hline Power $\left(\mathrm{nV}^{2}\right)$ & $5.95 \pm 1.71$ & $5.42 \pm 1.15$ & $7.26 \pm 3.52$ & 0.85 \\
\hline Peak frequency $(\mathrm{Hz})$ & $0.43 \pm 0.01$ & $0.47 \pm 0.01$ & $0.44 \pm 0.01$ & 0.06 \\
\hline
\end{tabular}

RMS: root mean square. Data are presented as mean \pm standard error of the mean.

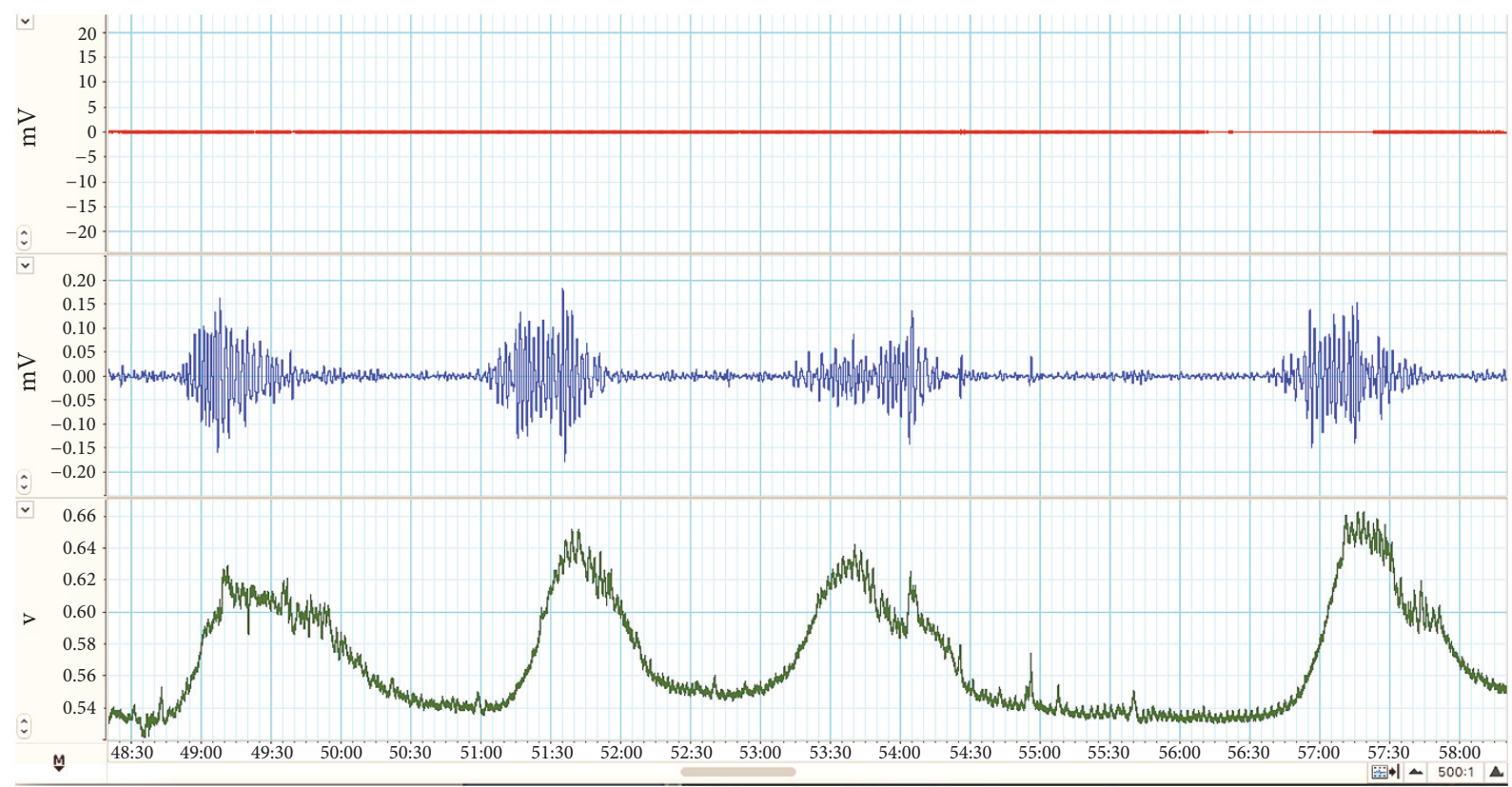

FIGURE 2: Representative EMG images. The representative recordings of ropivacaine group during the 1st stage of labor showing EMG signals from abdominal muscle (top tracings in red), uterine (middle tracings in blue), and TOCO signals (bottom tracings in green). A horizontal line above some of the bursts denotes bursts. The TOCO recorded signals (green tracings) correspond to TOCO recorded uterine contractions and occur at about the same time as EMG bursts. 


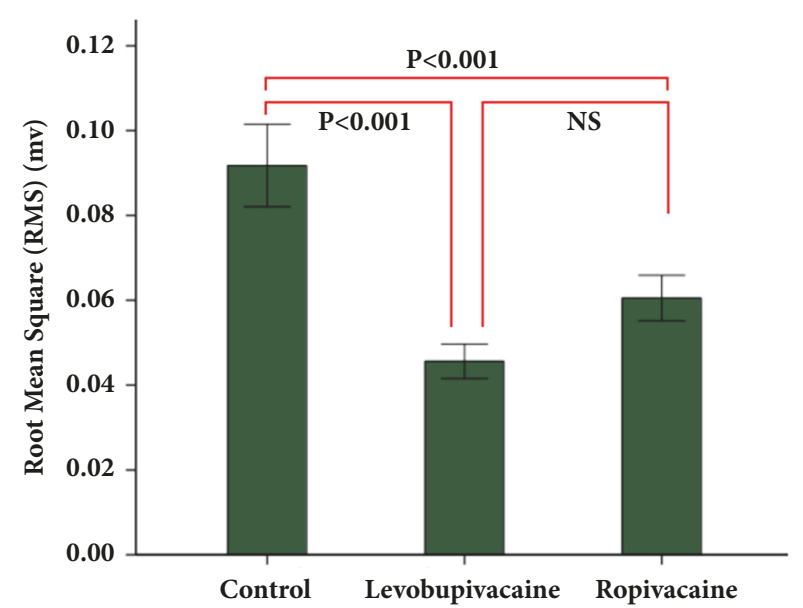

(a)

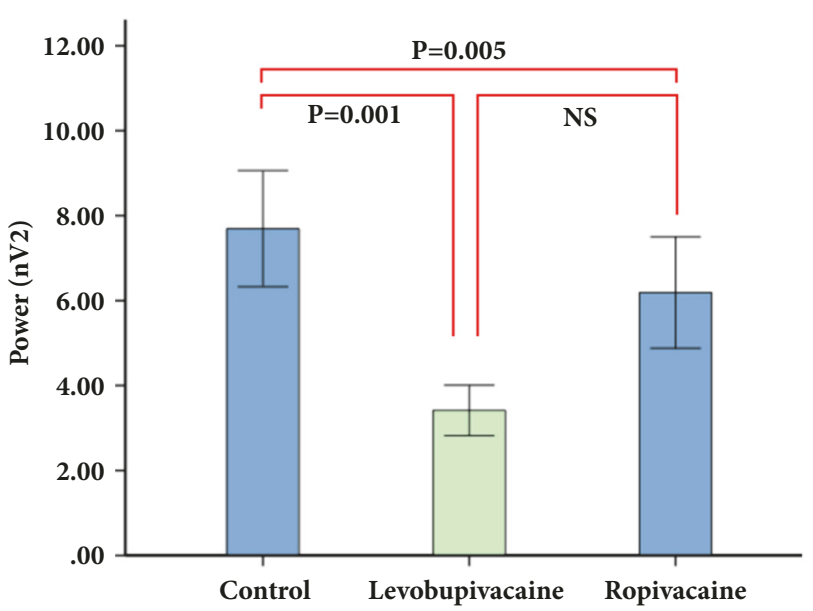

(b)

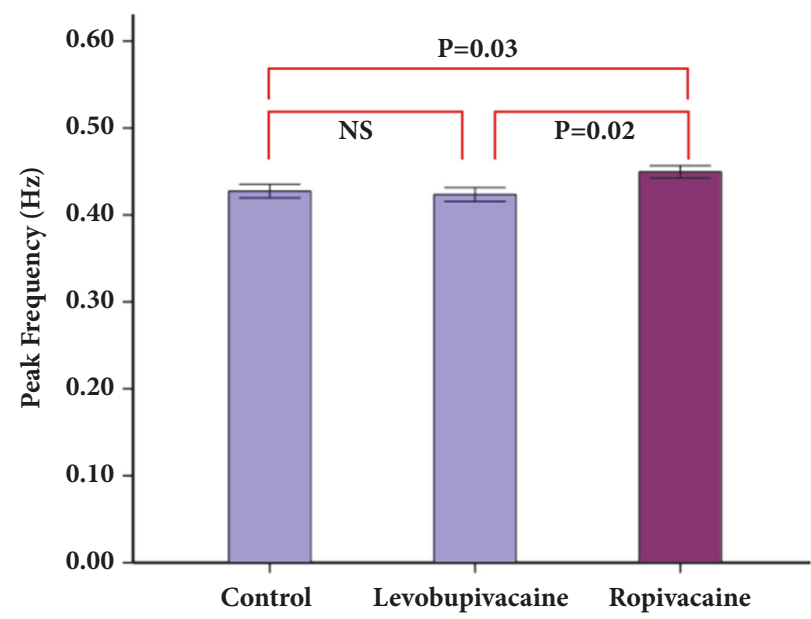

(c)

FIGURE 3: RMS, power, and peak frequency of EMG in the three groups. (a) RMS. (b) Power. (c) Peak frequency.

In 1984, bupivacaine $(0.75 \%$ solution) was reported to cause a number of obstetric deaths due to its cardiotoxicity. Then, the search for a safe as well as long-acting local anesthetic was launched. Two L-isomeric anesthetic agents, ropivacaine and levobupivacaine, have been developed. These two local anesthetics are better alternatives for epidural labor analgesia with less cardiac and neurological toxicity compared to bupivacaine $[22,23]$.

Ropivacaine is less likely to cause motor blockade and neurotoxicity due to its relatively low lipophilic capacity and resistance to penetrating readily into the myelinated nerve fibers [24, 25]. However, the superiority of ropivacaine over levobupivacaine for epidural labor analgesia remains controversial. Several studies indicated that ropivacaine and levobupivacaine have similar sensory and motor blocking effects in epidural labor analgesia [26-28], while another study showed that ropivacaine produced a similar level of analgesia as levobupivacaine but a significantly lower level of motor block [29]. In experimental studies, controversies regarding the effects of local anesthetics on myometrial contractility also exist [30-32]. In our study, the VAS pain scores of the ropivacaine group were comparable to those of the levobupivacaine group. Both levobupivacaine and ropivacaine, with a low concentration of $0.0625 \%$, produced good analgesic effects. These effects were mainly due to the use of a pulsed electronic infusion pump in the PCEA protocol through which the local anesthetics were administered and tailored by each individual patient.

Regarding uterine EMG activities, the values of RMS and power were significant higher in the ropivacaine group than in the levobupivacaine group. In addition, uterine EMG activities in the ropivacaine group were similar to those in the control group. Consistent with the findings on EMG, patients in the ropivacaine and control groups had a similar duration of the first stage of labor, while those in the levobupivacaine group had a significantly longer labor duration. These results were also consistent with our previous finding that levobupivacaine suppressed uterine EMG and prolonged the first stage of labor [17]. Moreover, the need for oxytocin administration was lower in the ropivacaine group compared with the levobupivacaine group. At different time points from 30 to $120 \mathrm{~min}$, the uterine EMG activity did not differ 
significantly in the ropivacaine group compared with the other groups. Taken together, the use of ropivacaine resulted in a less inhibitory effect on uterine contractility and labor progress than levobupivacaine.

There are several limitations to this study. First is the study design. This is a prospective cohort study rather than a randomized trial, and thus a potential bias may exist. Second, as previously reported, deep motor blockade caused by local anesthetics also prolongs the second stage of labor by reducing voluntary maternal expulsive forces [24]. However, in this study, we only investigated the EMG activities during the first stage. We did not evaluate the motor block effect of ropivacaine in the second stage of labor too. Another limitation was the lack of pain assessment after $30 \mathrm{~min}$. Further prospective studies are needed to strengthen these findings and the benefits of ropivacaine in epidural labor analgesia.

\section{Conclusions}

In conclusion, this is the first study to demonstrate that PCEA with ropivacaine leads to a better analgesic satisfaction without adverse effects on uterine EMG activities or obstetric outcomes during the first stage of labor compared to levobupivacaine. To obtain the best epidural anesthetic effect and obstetric outcomes without adverse effects, the optimal PCEA regimen for pain relief during labor requires further investigations.

\section{Data Availability}

The data used to support the findings of this study are included within the article.

\section{Conflicts of Interest}

The authors declare that they have no conflicts of interest.

\section{References}

[1] J. A. Thorp, D. H. Hu, R. M. Albin et al., "The effect of intrapartum epidural analgesia on nulliparous labor: A randomized, controlled, prospective trial," American Journal of Obstetrics \& Gynecology, vol. 169, no. 4, pp. 851-858, 1993.

[2] J. M. Alexander, M. J. Lucas, S. M. Ramin, D. D. McIntire, and K. J. Leveno, "The course of labor with and without epidural analgesia," American Journal of Obstetrics \& Gynecology, vol. 178, no. 3, pp. 516-520, 1998.

[3] E. Lieberman, K. Davidson, A. Lee-Parritz, and E. Shearer, "Changes in fetal position during labor and their association with epidural analgesia," Obstetrics \& Gynecology, vol. 105, no. 5, part 1, pp. 974-982, 2005.

[4] M. Anim-Somuah, R. M. Smyth, and L. Jones, "Epidural versus non-epidural or no analgesia in labour," Cochrane Database of Systematic Reviews, vol. 12, Article ID CD000331, 2011.

[5] B. L. Leighton and S. H. Halpern, "The effects of epidural analgesia on labor, maternal, and neonatal outcomes: a systematic review," American Journal of Obstetrics \& Gynecology, vol. 186, supplement 5, pp. S69-S77, 2002.
[6] P. Hincz, L. Podciechowski, M. Grzesiak, W. Horzelski, and J. Wilczyński, "Epidural analgesia during labour: a retrospective cohort study on its effects on labour, delivery and neonatal outcome," Polish Gynaecology, vol. 85, no. 12, pp. 923-928, 2014.

[7] E. R. Newton, B. C. Schroeder, K. G. Knape, and B. L. Bennett, "Epidural analgesia and uterine function," Obstetrics \& Gynecology, vol. 85, no. 5, pp. 749-755, 1995.

[8] G. Willdeck-Lund, G. Lindmark, and B. A. Nilsson, "Effect of Segmental Epidural Analgesia upon the Uterine Activity with Special Reference to the Use of Different Local Anaesthetic Agents," Acta Anaesthesiologica Scandinavica, vol. 23, no. 6, pp. 519-528, 1979.

[9] R. A. Fanning, D. P. Campion, C. B. Collins et al., "A comparison of the inhibitory effects of bupivacaine and levobupivacaine on isolated human pregnant myometrium contractility," Anesthesia \& Analgesia, vol. 107, no. 4, pp. 1303-1307, 2008.

[10] J. A. Katz, P. O. Bridenbaugh, D. C. Knarr, S. H. Helton, and D. D. Denson, "Pharmacodynamics and pharmacokinetics of epidural ropivacaine in humans," Anesthesia \& Analgesia, vol. 70, no. 1, pp. 16-21, 1990.

[11] A. Ahirwar, R. Prakash, B. B. Kushwaha et al., "Patient controlled epidural labour analgesia (PCEA): A comparison between ropivacaine, ropivacaine-fentanyl and ropivacaine-clonidine," Journal of Clinical and Diagnostic Research, vol. 8, no. 8, pp. GC09-GC13, 2014.

[12] X. Wang, S. Xu, X. Qin et al., "Comparison between the use of ropivacaine alone and ropivacaine with sufentanil in epidural labor analgesia," Medicine (United States), vol. 94, no. 43, Article ID e1882, 2015.

[13] G. Wolfs and H. Rottinghuis, "Electrical and mechanical activity of the human uterus during labour," Archiv für Gynäkologie, vol. 208, no. 4, pp. 373-385, 1970.

[14] W. L. Maner and R. E. Garfield, "Identification of human term and preterm labor using artificial neural networks on uterine electromyography data," Annals of Biomedical Engineering, vol. 35, no. 3, pp. 465-473, 2007.

[15] C. Rabotti and M. Mischi, "Propagation of electrical activity in uterine muscle during pregnancy: A review," Acta Physiologica, vol. 213, no. 2, pp. 406-416, 2015.

[16] X. Qian, P. Li, S.-Q. Shi, R. E. Garfield, and H. Liu, "Simultaneous Recording and Analysis of Uterine and Abdominal Muscle Electromyographic Activity in Nulliparous Women during Labor," Reproductive Sciences, vol. 24, no. 3, pp. 471-477, 2017.

[17] Y. Ye, X. Song, L. Liu et al., "Effects of Patient-Controlled Epidural Analgesia on Uterine Electromyography during Spontaneous Onset of Labor in Term Nulliparous Women," Reproductive Sciences, vol. 22, no. 11, pp. 1350-1357, 2015.

[18] R. E. Garfield, W. L. Maner, L. B. MacKay, D. Schlembach, and G. R. Saade, "Comparing uterine electromyography activity of antepartum patients versus term labor patients," American Journal of Obstetrics \& Gynecology, vol. 193, no. 1, pp. 23-29, 2005.

[19] H. Eswaran, H. Preissl, J. D. Wilson, P. Murphy, and C. L. Lowery, "Prediction of labor in term and preterm pregnancies using non-invasive magnetomyographic recordings of uterine contractions," American Journal of Obstetrics \& Gynecology, vol. 190, no. 6, pp. 1598-1603, 2004.

[20] T. Y. Euliano, D. Marossero, M. T. Nguyen, N. R. Euliano, J. Principe, and R. K. Edwards, "Spatiotemporal electrohysterography patterns in normal and arrested labor," American Journal of Obstetrics \& Gynecology, vol. 200, no. 1, pp. 54-el, 2009. 
[21] X. Qian, P. Li, S.-Q. Shi, R. E. Garfield, and H. Liu, "Uterine and Abdominal Muscle Electromyographic Activities in Control and PCEA-Treated Nulliparous Women during the Second Stage of Labor," Reproductive Sciences, vol. 24, no. 8, pp. 12141220, 2017.

[22] G. Lyons and F. Reynolds, "Toxicity and safety of epidural local anaesthetics," International Journal of Obstetric Anesthesia, vol. 10, no. 4, pp. 259-262, 2001.

[23] G. Kuthiala and G. Chaudhary, "Ropivacaine: a review of its pharmacology and clinical use," Indian Journal of Anaesthesia, vol. 55, no. 2, pp. 104-110, 2011.

[24] R. Lin, Y. Tao, Y. Yu et al., "Intravenous Remifentanil versus Epidural Ropivacaine with Sufentanil for Labour Analgesia: A Retrospective Study," PLoS ONE, vol. 9, no. 11, p. el12283, 2014.

[25] G. C. Meister, R. D’Angelo, M. Owen, K. E. Nelson, and R. Gaver, "A comparison of epidural analgesia with $0.125 \%$ ropivacaine with fentanyl versus $0.125 \%$ bupivacaine with fentanyl during labor," Anesthesia \& Analgesia, vol. 90, no. 3, pp. 632-637, 2000.

[26] B.-S. Lv, W. Wang, Z.-Q. Wang et al., "Efficacy and safety of local anesthetics bupivacaine, ropivacaine and levobupivacaine in combination with sufentanil in epidural anesthesia for labor and delivery: A meta-analysis," Current Medical Research and Opinion, vol. 30, no. 11, pp. 2279-2289, 2014.

[27] LZ. Wang, XY. Chang, X. Liu, and Hu., “Tang BL: Comparison of bupivacaine, ropivacaine and levobupivacaine with sufentanil for patient-controlled epidural analgesia during labor: a randomized clinical trial," in Chin Med J (Engl), pp. 123-178, 123, 178-183, 2010.

[28] N. Sah, M. Vallejo, A. Phelps, H. Finegold, G. Mandell, and S. Ramanathan, "Efficacy of ropivacaine, bupivacaine, and levobupivacaine for labor epidural analgesia," Journal of Clinical Anesthesia, vol. 19, no. 3, pp. 214-217, 2007.

[29] H. J. Lacassie, A. S. Habib, H. P. Lacassie, and M. O. Columb, "Motor Blocking Minimum Local Anesthetic Concentrations of Bupivacaine, Levobupivacaine, and Ropivacaine in Labor," Regional Anesthesia and Pain Medicine, vol. 32, no. 4, pp. 323329, 2007.

[30] D. O. Fauza, D. S. Kohane, E. B. Beeuwkes, N. Clayton, and T. J. Maher, "Local anesthetics inhibit uterine activity in vitro: Possible application on preterm labor prevention and treatment," Fetal Diagnosis and Therapy, vol. 18, no. 5, pp. 292-296, 2003.

[31] G. Arici, B. Karsli, N. Kayacan, and M. Akar, "The effects of bupivacaine, ropivacaine and mepivacaine on the contractility of rat myometrium," International Journal of Obstetric Anesthesia, vol. 13, no. 2, pp. 95-98, 2004.

[32] J.-S. Wei, Z.-B. Jin, Z.-Q. Yin et al., "Effects of local anesthetics on contractions of pregnant and non-pregnant rat myometrium in vitro," Acta Physiologica Hungarica, vol. 101, no. 2, pp. 228$235,2014$. 


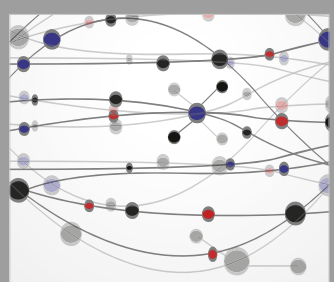

The Scientific World Journal
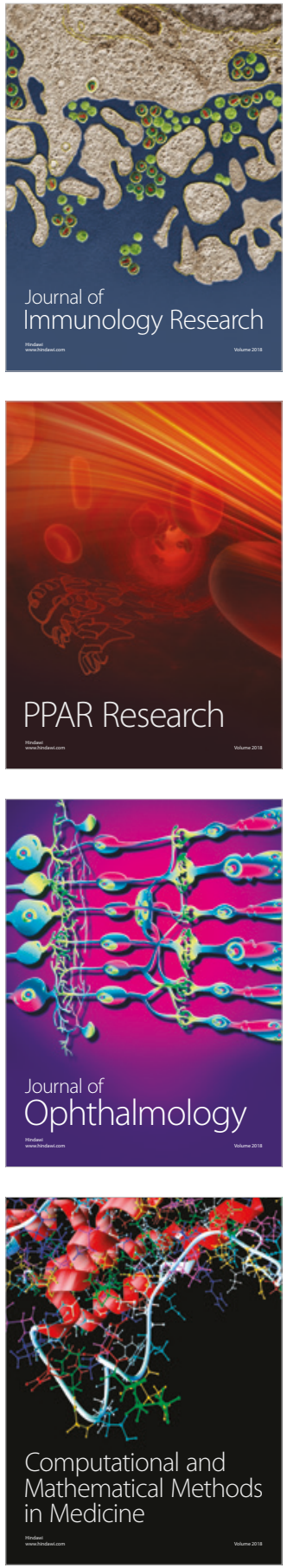

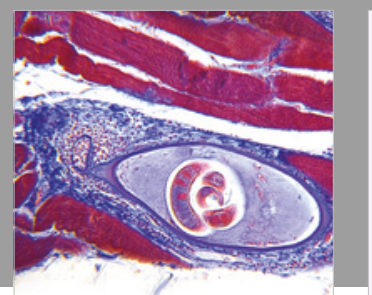

Gastroenterology Research and Practice

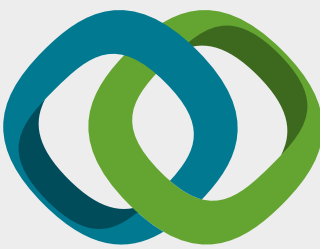

\section{Hindawi}

Submit your manuscripts at

www.hindawi.com
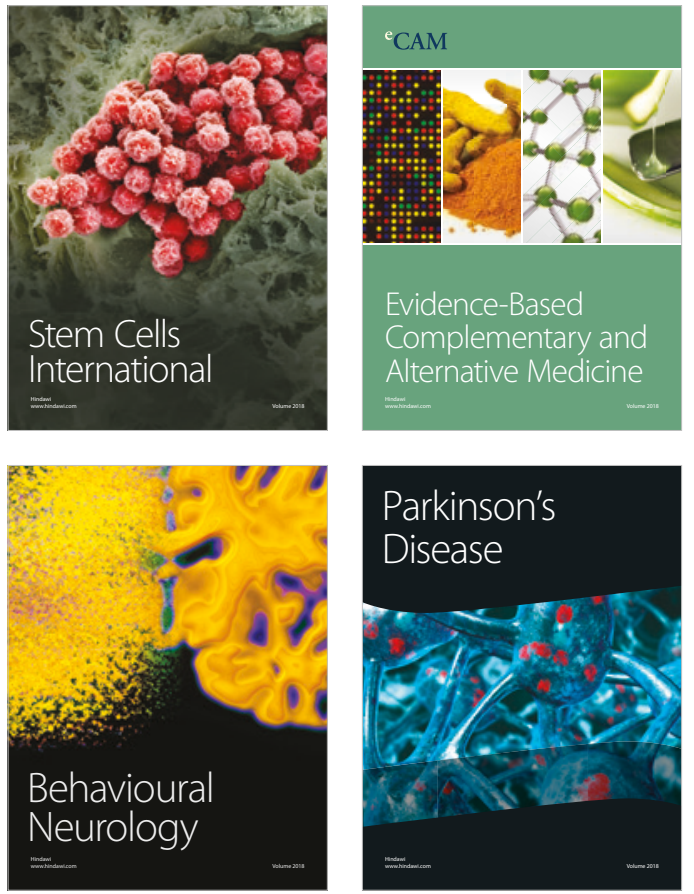

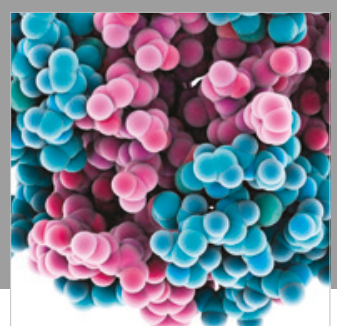

ournal of

Diabetes Research

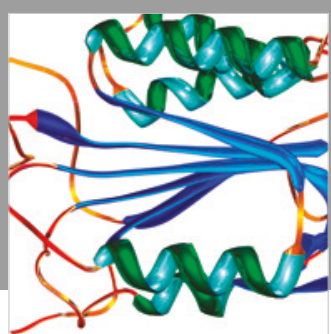

Disease Markers
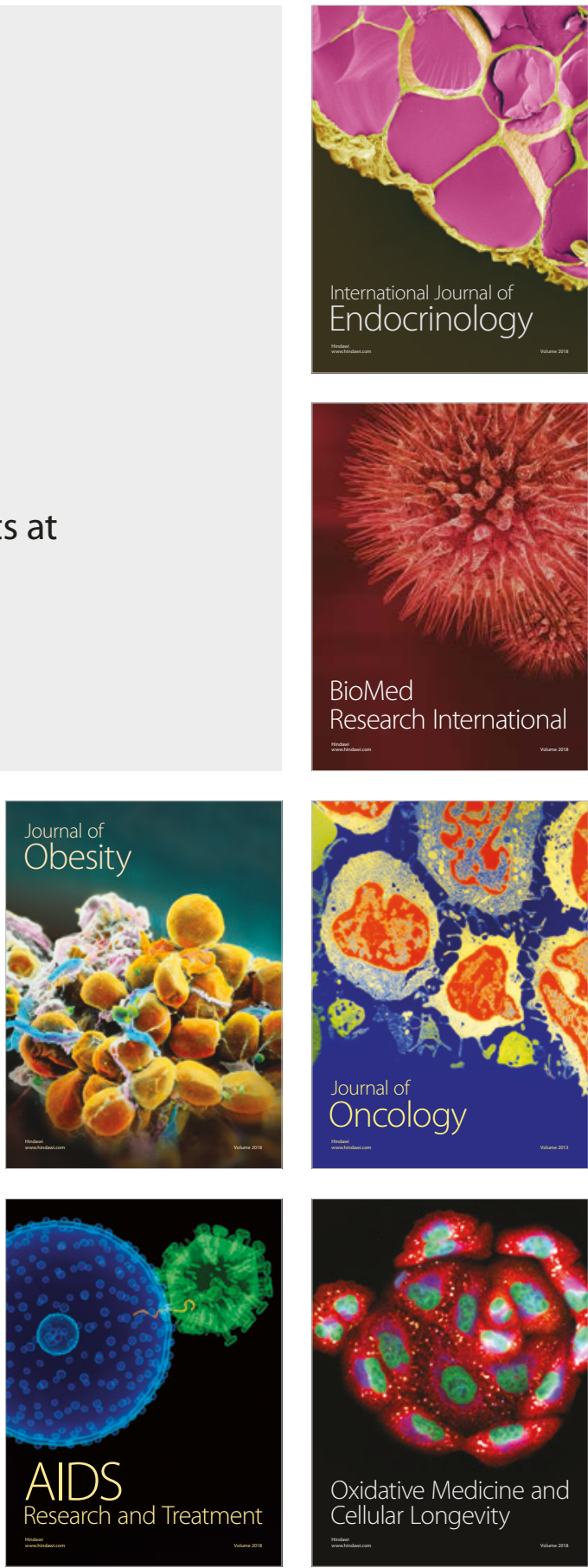